\title{
Tendência do estado nutricional de gestantes adolescentes beneficiárias do programa de transferência condicionada de renda brasileiro Bolsa Família no período 2008-2018
}

\author{
Trend of the nutritional status of pregnant adolescent beneficiaries \\ of the Brazilian Bolsa Família conditional cash transfer program \\ in the $2008-2018$ period
}

\author{
André Eduardo da Silva Júnior (https://orcid.org/0000-0002-1501-171X) ${ }^{1}$ \\ Mateus de Lima Macena (https://orcid.org/0000-0002-7168-9605) ${ }^{2}$ \\ Laís Gomes Lessa Vasconcelos (https://orcid.org/0000-0002-9445-1966) ${ }^{2}$ \\ Nykholle Bezerra Almeida (https://orcid.org/0000-0002-8536-3972) ${ }^{2}$ \\ Dafiny Rodrigues Silva Praxedes (https://orcid.org/0000-0002-5345-3869) ${ }^{2}$ \\ Isabele Rejane de Oliveira Maranhão Pureza (https://orcid.org/0000-0002-0652-429X) ${ }^{1}$ \\ Nassib Bezerra Bueno (https://orcid.org/0000-0002-3286-0297) 1,2 \\ Ana Paula Grotti Clemente (https://orcid.org/0000-0003-2315-5980) ${ }^{2}$
}

${ }^{1}$ Programa de PósGraduação em Nutrição, Escola Paulista de Medicina, Universidade Federal de São Paulo. R. Botucatu s/n, Vila Clementino. 04023 062 São Paulo SP Brasil. andreeduardojr@

hotmail.com

${ }^{2}$ Faculdade de Nutrição,

Universidade Federal de

Alagoas. Maceió AL Brasil.
Abstract This ecological study aimed to analyze the trend of the nutritional status of pregnant adolescent beneficiaries of the Brazilian Bolsa Familia conditional cash transfer program in the 2008-2018 period. We evaluated secondary data of pregnant adolescent beneficiaries of the Bolsa Familia Program from January 2008 to December 2018, extracted from the public reports of the WEB Food and Nutrition Surveillance System. We accessed the monitoring reports on the consolidated public-access health conditionalities of the Bolsa Familia Program, always considering the second validity. An annual variation of $-1.2 \%$ (95\%CI: $[-1.6 ;-0.8] p<0.01)$ was observed in the prevalence of underweight in Brazil in the studied sample. The prevalence of overweight and obesity in the country had annual variations of $2.9 \%$ (95\%CI: $[2.0 ; 3.7] p<0.01)$ and $7.5 \%$ (95\%CI: [5.7; 9.3] $p<0.01$ ), respectively. We conclude by saying, that, in the evaluated period, the prevalence of underweight among pregnant adolescent beneficiaries of the Bolsa Familia Program showed a decreasing trend, while the prevalence of overweight and obesity increased throughout Brazil.

Key words Pregnancy in adolescence, Nutrition assessment, Nutritional surveillance, Health information systems
Resumo Trata-se de um estudo ecológico que objetivou analisar a tendência do estado nutricional de gestantes adolescentes beneficiárias do programa brasileiro de transferência condicionada de renda, Bolsa Família, no período 2008-2018. Foram avaliados dados secundários de gestantes adolescentes beneficiárias do Programa Bolsa Família no período de janeiro de 2008 a dezembro de 2018, extraídos dos relatórios públicos do Sistema de Vigilância Alimentar e Nutricional WEB. Foram consultados os relatórios de acompanhamento das condicionalidades de saúde consolidados de acesso público do Programa Bolsa Família, sempre considerando a $2^{a}$ vigência. Observou-se uma variação anual de -1,2\% (IC95\%: [-1,6; -0,8] $p<0,01)$ na prevalência de baixo peso na amostra estudada. Com relação as prevalências de sobrepeso e obesidade no país, observou-se variações anuais de 2,9\% (IC95\%: [2,0; 3,7] $p<0,01)$ e 7,5\% (IC95\%: [5,7; 9,3] $p<0,01)$, respectivamente. Conclui-se que, no período avaliado, a prevalência de baixo peso entre as gestantes adolescentes beneficiárias do Programa Bolsa Família apresentou uma tendência decrescente, ao passo que as prevalências de sobrepeso e obesidade apresentam uma tendência crescente em todo o Brasil.

Palavras-chave Gravidez na adolescência, Avaliação nutricional, Vigilância alimentar e nutricional, Sistemas de informação em saúde 


\section{Introdução}

A gravidez na adolescência é um problema de saúde pública mundial devido às suas consequências biológicas, psicológicas, econômicas, familiares e educacionais, que podem reverberar sobre os indicadores sociais, econômicos e de saúde de um país' ${ }^{1}$. De acordo com a Organização Mundial de Saúde (OMS), a adolescência é o período de transição entre a infância e a idade adulta, com início aos 10 e se estendendo até os 19 anos de idade ${ }^{2}$. Estimativas da OMS, apontam que 16 milhões de adolescentes, com idade entre 15 e 19 anos, engravidam por ano, o que equivale a cerca de $11 \%$ do total de nascimentos em todo o mundo ${ }^{3}$. No Brasil, estima-se que a prevalência de gestantes nessa mesma faixa etária é de $11,8 \%{ }^{4}$. Segundo dados do relatório publicado pela Organização das Nações Unidas (ONU), a taxa de gravidez na adolescência no Brasil é de 68,4 nascimentos para cada 1.000 adolescentes, superando as taxas da América Latina e Caribe, e mundial (65,5 e 46,0 nascimentos para cada 1.000 adolescentes, respectivamente) $)^{5}$.

A gestação nesta fase do ciclo da vida aumenta ainda mais as necessidades energéticas e nutricionais que já são mais elevadas, devido ao fato de ser uma fase caracterizada pela finalização do processo de crescimento estatural, aumento do peso corporal, acréscimo de massa óssea, maturação dos órgãos sexuais, alterações na composição corporal, o que pode provocar competição por nutrientes entre a mãe e o feto e promover diminuição do crescimento linear das mães ${ }^{6,7}$. Ademais, os riscos de desfechos adversos para o binômio mãe-filho nessa faixa etária vêm sendo relatados na literatura científica, sendo os principais: o baixo peso ao nascer, prematuridade, natimorto, pré-eclâmpsia, sentimento de isolamento social, depressão materna e atraso ou negligência da educação materna ${ }^{8-10}$.

A ocorrência de gestações na adolescência parece sofrer importante influência de questões sociais e econômicas. A OMS estimou que cerca de 95\% dos partos de mães com idade entre 15 e 19 anos acontecem nos países em desenvolvimento ${ }^{3}$. Esta condição é impulsionada pela pobreza, baixa escolaridade, exclusão social, sexual e de gênero, falta de oportunidades de emprego, e união/casamento precoce ${ }^{5,11}$. Diante desse contexto, a gravidez na adolescência contribui para a manutenção dos ciclos intergeracionais da pobreza, exclusão e marginalização, caracterizados pelo maior risco de pobreza e piores desfechos relacionados à saúde nos filhos de mães adolescentes ${ }^{5}$.
Os programas de transferências de renda com condicionalidades, a exemplo do Programa Bolsa Família (PBF) no Brasil, são reconhecidos mundialmente por amenizar a pobreza dos seus beneficiários, aumentar a utilização dos serviços de saúde e melhorar os indicadores de saúde de crianças e adultos ${ }^{12-14}$. O PBF pode ser considerado uma das mais importantes iniciativas do governo brasileiro no combate à pobreza no país. Resultado da unificação dos programas de transferência de renda existentes, foi implantado pelo Governo Federal em 2003, por meio da Medida Provisória no 132, que posteriormente foi convertida na Lei $\mathrm{n}^{\circ} 10.836$, de 9 de janeiro de 2004. O programa cresceu e se tornou o maior do mundo, pagando mais de $\mathrm{R} \$ 31$ bilhões a 14 milhões de beneficiários somente em $2019^{15}$.

O PBF consiste em um programa de transferência direta de renda com condicionalidades que visa o alívio imediato da pobreza para que, a longo prazo, haja a melhora no desenvolvimento do capital humano através de maior acesso à educação, saúde e promoção da segurança alimentar. Entre as condicionalidades do PBF na área da saúde para as gestantes está o dever do comparecimento às consultas de pré-natal com o acompanhamento do estado nutricional, vacinação, conforme calendário preconizado pelo Ministério da Saúde e participação nas atividades educativas sobre aleitamento materno, orientações para alimentação saudável da gestante e preparo para o parto. Com o intuito de aumentar a proteção à mãe e à criança e elevar a renda familiar, o PBF estabelece que famílias beneficiárias do PBF com gestantes identificadas nos estabelecimentos de atenção à saúde, recebam nove parcelas mensais do Benefício Variável Gestante (BVG). Com isso, o programa busca além do fornecimento monetário, tornar-se um instrumento de promoção de equidade no acesso a direitos sociais básicos ${ }^{14,16,17}$.

Contudo, um estudo de revisão que avaliou os impactos do PBF na promoção da segurança alimentar entre os beneficiários (não selecionando faixa etária específica), através da avaliação do gasto financeiro com alimentação, consumo alimentar, níveis de hemoglobina, insegurança alimentar e dados antropométricos, observou o seguinte paradoxo: ao mesmo tempo em que o programa promove um aumento no acesso aos alimentos em termos de quantidade, não necessariamente tal aumento é acompanhado por um aumento da qualidade nutricional dos alimentos adquiridos ${ }^{18}$. Lignani et al. ${ }^{19}$ observaram ainda que com o aumento do poder de compra das famílias beneficiárias do programa houve um au- 
mento no consumo de alimentos industrializados com alta densidade energética, possivelmente associado ao menor custo destes alimentos. Estes fatos podem contribuir para o aumento da prevalência de desvios nutricionais nos beneficiários do programa. Assim, o objetivo deste trabalho foi analisar a tendência do estado nutricional de gestantes adolescentes beneficiárias do PBF, no período 2008-2018.

\section{Métodos}

Trata-se de um estudo ecológico, em que foram avaliados os dados secundários das gestantes adolescentes beneficiárias do PBF no período de janeiro de 2008 a dezembro de 2018, extraídos dos relatórios públicos do Sistema de Vigilância Alimentar e Nutricional (SISVAN) Web (http:// sisaps.saude.gov.br/sisvan/relatoriopublico/index, acessado em 15/12/2019). Para a geração dos relatórios foram utilizados os filtros "gestantes", "adolescentes", e para o sistema de origem dos dados "sistema de gestão do bolsa família (DATASUS)" e exibidos os dados consolidados do Brasil, além de estratificados de acordo com as Unidades da Federação (UF) e as macrorregiões Norte, Nordeste, Centro-Oeste, Sudeste e Sul do país.

O estado nutricional das gestantes disponibilizados no SISVAN Web, são classificados de acordo com o gráfico de Índice de Massa Corporal (IMC) segundo a semana gestacional proposto por Atalah et al. ${ }^{20}$. Neste instrumento são propostas quatro categorias do estado nutricional: baixo peso, eutrofia, sobrepeso e obesidade. Destaca-se que esta classificação do estado nutricional não é específica para gestantes adolescentes, mas segundo as orientações para coleta em serviços de saúde do Ministério da Saúde brasileiro, esta pode ser usada neste público desde que a interpretação dos resultados seja flexível e se considere a especificidade do grupo em questão ${ }^{21}$.

Para determinar o percentual de cobertura de acompanhamento nutricional de gestantes beneficiarias do PBF, foram consultados os relatórios de acompanhamento das condicionalidades de saúde consolidados de acesso público do PBF, sempre considerando a $2^{\text {a }}$ vigência (https://bfa. saude.gov.br/relatorio/consolidado e http://bolsafamilia.datasus.gov.br/w3c/bfa_relconsol.asp, acessados em 16/12/2019). Esta cobertura leva em consideração o número de gestantes beneficiárias do programa que foram localizadas por equipes de saúde e o número de gestantes que ti- veram dados nutricionais incluídos nos sistemas de informação.

O presente estudo não foi submetido para avaliação do sistema dos Comitês de Ética em Pesquisa e da Comissão Nacional de Ética em Pesquisa (CEP/CONEP), tendo em vista que se trata de uma pesquisa que utiliza bancos de dados de domínio público, como dispõe a Resolução no 510, de 07 de abril de 2019, do Conselho Nacional de Saúde (CNS).

Após a extração, os dados foram tabulados em planilhas eletrônicas por meio de dupla entrada independente e, posteriormente, foi realizada a validação dos dados. A variação relativa da prevalência dos desvios nutricionais no período foi calculada usando a seguinte fórmula: variação relativa $=((\mathrm{P} 2-\mathrm{P} 1) / \mathrm{P} 1)^{*} 100$, onde $\mathrm{P} 2$ correspondia à prevalência do desvio nutricional no ano 2018 e P1 à prevalência no ano 2008. As prevalências foram apresentadas para cada ano do período avaliado (2008-2018), assim como os intervalos de confiança (IC) para proporções populacionais.

Na sequência, para avaliar a tendência da série temporal, utilizou-se a análise linear generalizada de Prais-Winsten ${ }^{22}$. No modelo, a prevalência de estado nutricional (baixo peso, sobrepeso e obesidade) foi a variável dependente e os anos de avaliação, a variável independente. Aplicou-se o teste de Durbin-Watson para verificar a autocorrelação da série. Utilizou-se o método sugerido por Antunes e Cardoso ${ }^{22}$. Primeiramente, os valores das prevalências dos estados nutricionais foram logaritimizados. Depois, aplicou-se o modelo autorregressivo de Prais-Winsten, estimando os valores beta, beta mínimo e beta máximo. Com estes valores, calculou-se a taxa de variação anual e seus respectivos IC95\%, que podem ser interpretados como crescente (taxa de variação positiva), estacionária (quando não existe diferença estatística) e decrescente (taxa de variação negativa). Para os níveis de significância estatística, foram adotados valores de alfa de 5\%. Todas as análises estatísticas foram conduzidas no pacote estatístico R ( $R$ Foundation for Statistical Computing, Vienna, Áustria).

\section{Resultados}

O número de observações anuais de gestantes adolescentes passou de 11.835 para 92.577 , um aumento de 682,2\%, entre os anos de 2008 e 2018. Com relação ao estado nutricional das gestantes adolescentes, observou-se que em 2008, o Brasil 
apresentou prevalências de $37,8 \%$ de baixo peso, $46,9 \%$ de eutrofia, $12,1 \%$ de sobrepeso e $3,3 \%$ de obesidade. Em 2018, foram observadas prevalências de $33,1 \%$ de baixo peso, $43,7 \%$ de eutrofia, $16,4 \%$ de sobrepeso e $6,8 \%$ de obesidade no país, com variações relativas de sobrepeso e obesidade positivas em relação a 2008 (35,5 e 106,1\%, respectivamente). Em contrapartida, as observações de baixo peso apresentaram variação relativa negativa de $12,4 \%$. As demais prevalências de baixo peso, eutrofia, sobrepeso e obesidade, e variações relativas no Brasil e UF no período de 2008 e 2018 estão expostas na Tabela 1. As prevalências brutas dos desvios nutricionais (baixo peso, sobrepeso e obesidade) nos anos de 2008 e 2018 estão presentes graficamente na Figura 1.

$\mathrm{Na}$ Tabela 2, observa-se a relação de gestantes localizadas por equipes de saúde por região e gestantes com dados nutricionais incluídos nos sistemas de informação, na qual é verificado que em 2008, as regiões Sudeste e Norte apresentaram o menor $(46,9 \%)$ e o maior $(66,8 \%)$ percentual de acompanhamento, respectivamente. Já em 2018, o Brasil mostrou um acompanhamento dos dados nutricionais de $87,3 \%$ gestantes adolescentes localizadas, sendo este maior do que $80 \%$ em todas as cinco regiões do país.

A Tabela 3 apresenta as análises de tendência das prevalências de baixo peso, sobrepeso e obesidade em gestantes adolescentes beneficiárias do PBF no Brasil e em suas macrorregiões. Observou-se uma tendência decrescente na prevalência de baixo peso no Brasil com variação anual de $-1,2 \%$ (IC95\%: $[-1,6 ;-0,8] \mathrm{p}<0,01$ ), sendo a região Sul a que apresentou a maior variação anual $(-1,5 \%$; IC95\%: $[-2,1 ;-0,9] \mathrm{p}<0,01)$. Com relação a prevalência de sobrepeso, observou-se uma tendência crescente no país (2,9\%; IC95\%: $[2,0 ; 3,7] \mathrm{p}<0,01)$, destaca-se que a região Norte apresentou a maior variação anual entre as regiões $(4,1 \%$; IC95\%: $[3,1 ; 5,2] \mathrm{p}<0,01)$. As prevalências de obesidade também apresentaram tendência crescente no país (7,5\%; IC95\%: [5,7; 9,3] $\mathrm{p}<0,01)$, sendo as regiões Centro-Oeste $(10,2 \%$; IC95\%: $[5,3 ; 15,3] \mathrm{p}<0,01)$ e Nordeste $(10,1 \%$; IC95\%: $[7,8 ; 12,5] \mathrm{p}<0,01)$ as com as maiores variações anuais.

\section{Discussão}

O estudo verificou uma tendência decrescente na prevalência de baixo peso entre as gestantes adolescentes beneficiárias do PBF no período avaliado, ao mesmo tempo que a prevalência de sobre- peso e obesidade apresentou tendências crescentes em todas as macrorregiões do país. Com relação a cobertura do PBF, observamos um aumento no percentual de gestantes com dados nutricionais inseridos nos sistemas de informação no período avaliado. Apesar de não ter sido o foco do estudo comparar os dados de gestantes inseridos nos sistemas com a cobertura da Estratégia Saúde da Família (ESF), é admissível acreditar que este fato se encontre associado à ascensão da cobertura da ESF em o todo o território nacional ${ }^{23}$.

Os achados que apontam para a tendência crescente na prevalência de gestantes adolescentes com excesso de peso no Brasil e nas suas regiões, segue o mesmo padrão mundial em crianças, adolescentes e adultos ${ }^{24}$. Nossos resultados indicam que as condicionalidades e a transferência de renda realizadas atualmente pelo PBF, não foram suficientes para garantir o acesso a segurança alimentar e nutricional e reduzir os agravos nutricionais de adolescentes gestantes beneficiarias, necessitando de medidas intersetoriais voltadas às famílias e comunidade. Este aumento simultâneo da obesidade nos ciclos da vida concomitante a perpetuação das elevadas prevalências de baixo peso parece ser impulsionado por mudanças nos sistemas alimentares globais, fenômeno observado nos países de baixa e média renda decorrente do processo de transição alimentar que oferece mais alimentos processados, acessíveis e densos energeticamente ${ }^{24,25}$. Estudos descrevem que este fenômeno leva ao aumento do risco para as doenças crônicas não transmissíveis, mostrando um ciclo intergeracional de desvantagens, condição que culmina numa alta carga de morbidade, como o aumento de doenças cardiovasculares, diabetes mellitus tipo 2, câncer, osteoartrite e incapacidade de trabalho ${ }^{24,26,27}$. Nas mulheres, a exposição a esta dupla carga de desnutrição ao longo da vida pode levar à maiores complicações no parto ${ }^{27}$. Além disso, a obesidade na gestação pode aumentar o risco para diversas complicações maternas e infantis, como aborto espontâneo, diabetes mellitus gestacional, pré-eclâmpsia, tromboembolismo pós-parto, problemas no aleitamento materno, nascimento pré-maturo, natimorto, macrossomia e anomalias congênitas ${ }^{28}$.

Cumprir as condicionalidades do PBF significa, para o beneficiário, a garantia do exercício de seus direitos sociais, em especial os de saúde, educação e assistência social; acesso a esses serviços é um direito básico e condição fundamental para o rompimento do ciclo intergeracional da pobreza ${ }^{17}$. Os dados de acompanhamento pré-natal das gestantes beneficiárias do PBF são 
Tabela 1. Prevalência de baixo peso, eutrofia, sobrepeso e obesidade em gestantes adolescentes beneficiárias do Programa Bolsa Família no período 2008-2018, segundo unidades federativas do Brasil.

\begin{tabular}{|c|c|c|c|c|c|c|c|c|c|c|c|c|}
\hline \multirow[b]{2}{*}{ Local } & \multicolumn{3}{|c|}{ Baixo peso (\%) } & \multicolumn{3}{|c|}{ Eutrofia (\%) } & \multicolumn{3}{|c|}{ Sobrepeso (\%) } & \multicolumn{3}{|c|}{ Obesidade (\%) } \\
\hline & 2008 & 2018 & $\begin{array}{l}\text { Variação } \\
\text { relativa }^{a}\end{array}$ & 2008 & 2018 & $\begin{array}{l}\text { Variação } \\
\text { relativa }^{a}\end{array}$ & 2008 & 2018 & $\begin{array}{l}\text { Variação } \\
\text { relativa }^{a}\end{array}$ & 2008 & 2018 & $\begin{array}{l}\text { Variação } \\
\text { relativa }^{\text {a }}\end{array}$ \\
\hline \multicolumn{13}{|l|}{ Norte } \\
\hline Acre & 36,4 & 28,7 & $-21,2$ & 51,1 & 48,9 & $-4,3$ & 11,4 & 17,1 & 50,0 & 1,1 & 5,3 & 381,8 \\
\hline Amapá & 31,0 & 34,7 & 11,9 & 48,3 & 43,4 & $-10,1$ & 17,2 & 16,8 & $-2,3$ & 3,5 & 5,2 & 48,6 \\
\hline Amazonas & 36,4 & 34,3 & $-5,8$ & 49,3 & 46,8 & $-5,1$ & 10,8 & 14,8 & 37,0 & 3,5 & 4,1 & 17,1 \\
\hline Pará & 40,0 & 34,6 & $-13,5$ & 48,1 & 47,1 & $-2,1$ & 9,6 & 14,1 & 46,9 & 2,4 & 4,2 & 75,0 \\
\hline Rondônia & 45,1 & 39,7 & $-12,0$ & 43,7 & 39,1 & $-10,5$ & 8,5 & 15,9 & 87,1 & 2,8 & 5,3 & 89,3 \\
\hline Roraima & 33,3 & 27,5 & $-17,4$ & 58,3 & 51,8 & $-11,1$ & 4,2 & 15,9 & 278,6 & 4,2 & 4,7 & 11,9 \\
\hline Tocantins & 42,2 & 35,9 & $-14,9$ & 46,4 & 42,9 & $-7,5$ & 8,4 & 15,8 & 88,1 & 3,0 & 5,4 & 80,0 \\
\hline \multicolumn{13}{|l|}{ Nordeste } \\
\hline Alagoas & 38,0 & 35,1 & $-7,6$ & 47,5 & 42,7 & $-10,1$ & 10,6 & 15,4 & 45,3 & 4,0 & 6,8 & 70,0 \\
\hline Bahia & 42,6 & 37,3 & $-12,4$ & 45,2 & 42,7 & $-5,5$ & 10,2 & 14,3 & 40,2 & 2,0 & 5,8 & 190,0 \\
\hline Ceará & 40,6 & 32,3 & $-20,4$ & 45,3 & 42,6 & $-6,0$ & 11,8 & 18,1 & 53,4 & 2,3 & 7,0 & 204,3 \\
\hline Maranhão & 40,4 & 37,4 & $-7,4$ & 50,6 & 44,4 & $-12,3$ & 8,0 & 14,2 & 77,5 & 1,0 & 4,0 & 300,0 \\
\hline Paraíba & 34,2 & 32,1 & $-6,1$ & 48,6 & 44,0 & $-9,5$ & 14,4 & 16,9 & 17,4 & 2,9 & 7,0 & 141,4 \\
\hline Pernambuco & 36,5 & 33,3 & $-8,8$ & 48,1 & 44,5 & $-7,5$ & 13,0 & 15,8 & 21,5 & 2,5 & 6,5 & 160,0 \\
\hline Piauí & 41,5 & 36,9 & $-11,1$ & 45,0 & 42,5 & $-5,6$ & 11,9 & 15,1 & 26,9 & 1,6 & 5,6 & 250,0 \\
\hline Rio Grande do Norte & 35,6 & 30,2 & $-15,2$ & 46,7 & 42,5 & $-9,0$ & 13,8 & 19,3 & 39,9 & 4,0 & 8,1 & 102,5 \\
\hline Sergipe & 50,4 & 36,7 & $-27,2$ & 37,6 & 41,3 & 9,8 & 8,6 & 15,1 & 75,6 & 3,4 & 6,9 & 102,9 \\
\hline \multicolumn{13}{|l|}{ Centro-Oeste } \\
\hline Distrito Federal & 43,9 & 37,6 & $-14,4$ & 46,3 & 39,7 & $-14,3$ & 9,8 & 14,7 & 50,0 & 0,0 & 7,9 & - \\
\hline Goiás & 38,4 & 33,2 & $-13,5$ & 40,6 & 43,1 & 6,2 & 17,8 & 16,7 & $-6,2$ & 3,3 & 7,1 & 115,2 \\
\hline Mato Grosso & 36,0 & 35,0 & $-2,8$ & 46,7 & 40,8 & $-12,6$ & 14,2 & 16,4 & 15,5 & 3,1 & 7,8 & 151,6 \\
\hline Mato Grosso do Sul & 37,3 & 30,3 & $-18,8$ & 46,3 & 45,2 & $-2,4$ & 14,9 & 15,7 & 5,4 & 1,5 & 8,7 & 480,0 \\
\hline \multicolumn{13}{|l|}{ Sudeste } \\
\hline Espírito Santo & 37,4 & 32,3 & $-13,6$ & 43,3 & 42,0 & $-3,0$ & 15,6 & 17,7 & 13,5 & 3,8 & 8,1 & 113,2 \\
\hline Minas Gerais & 39,1 & 33,3 & $-14,8$ & 45,9 & 43,0 & $-6,3$ & 11,8 & 16,4 & 39,0 & 3,3 & 7,3 & 121,2 \\
\hline Rio de Janeiro & 38,1 & 32,8 & $-13,9$ & 48,1 & 40,2 & $-16,4$ & 11,4 & 18,2 & 59,0 & 2,4 & 8,8 & 266,7 \\
\hline São Paulo & 33,3 & 29,0 & $-12,9$ & 46,2 & 42,6 & $-7,8$ & 14,2 & 18,7 & 31,7 & 6,3 & 9,8 & 55,6 \\
\hline \multicolumn{13}{|l|}{ Sul } \\
\hline Paraná & 32,4 & 27,2 & $-16,0$ & 50,3 & 44,7 & $-11,1$ & 13,2 & 19,2 & 45,5 & 4,1 & 9,0 & 119,5 \\
\hline Rio Grande do Sul & 28,6 & 23,1 & $-19,2$ & 47,8 & 44,6 & $-6,7$ & 17,5 & 20,3 & 16,0 & 6,1 & 12,1 & 98,4 \\
\hline Santa Catarina & 29,6 & 21,7 & $-26,7$ & 51,6 & 49,4 & $-4,3$ & 13,6 & 19,1 & 40,4 & 5,2 & 9,8 & 88,5 \\
\hline Brasil & 37,8 & 33,1 & $-12,4$ & 46,9 & 43,7 & $-6,8$ & 12,1 & 16,4 & 35,5 & 3,3 & 6,8 & 106,1 \\
\hline
\end{tabular}

Fonte: Sistema de Vigilância Alimentar e Nutricional Web.

inseridos no SISVAN, que é um sistema de informação em saúde que tem como objetivo a geração de informações sobre a situação alimentar e nutricional da população, contribuindo para o conhecimento da natureza e da magnitude dos problemas de nutrição, identificando áreas geográficas, segmentos sociais e grupos populacionais de maior risco aos agravos nutricionais. Ressalta-se a importância da coleta adequada dos dados e da alimentação do sistema, para que este possa fornecer subsídios para formulação de políticas públicas que visem à melhoria dos padrões de consumo alimentar e do estado nutricional da população ${ }^{16}$.

A assistência pré-natal de qualidade é fundamental para a saúde materna e neonatal. Nesta perspectiva, a assistência nutricional composta por orientações nutricionais e acompanhamento contínuo do estado nutricional, contribui para o ganho de peso ideal durante a gestação evitando 

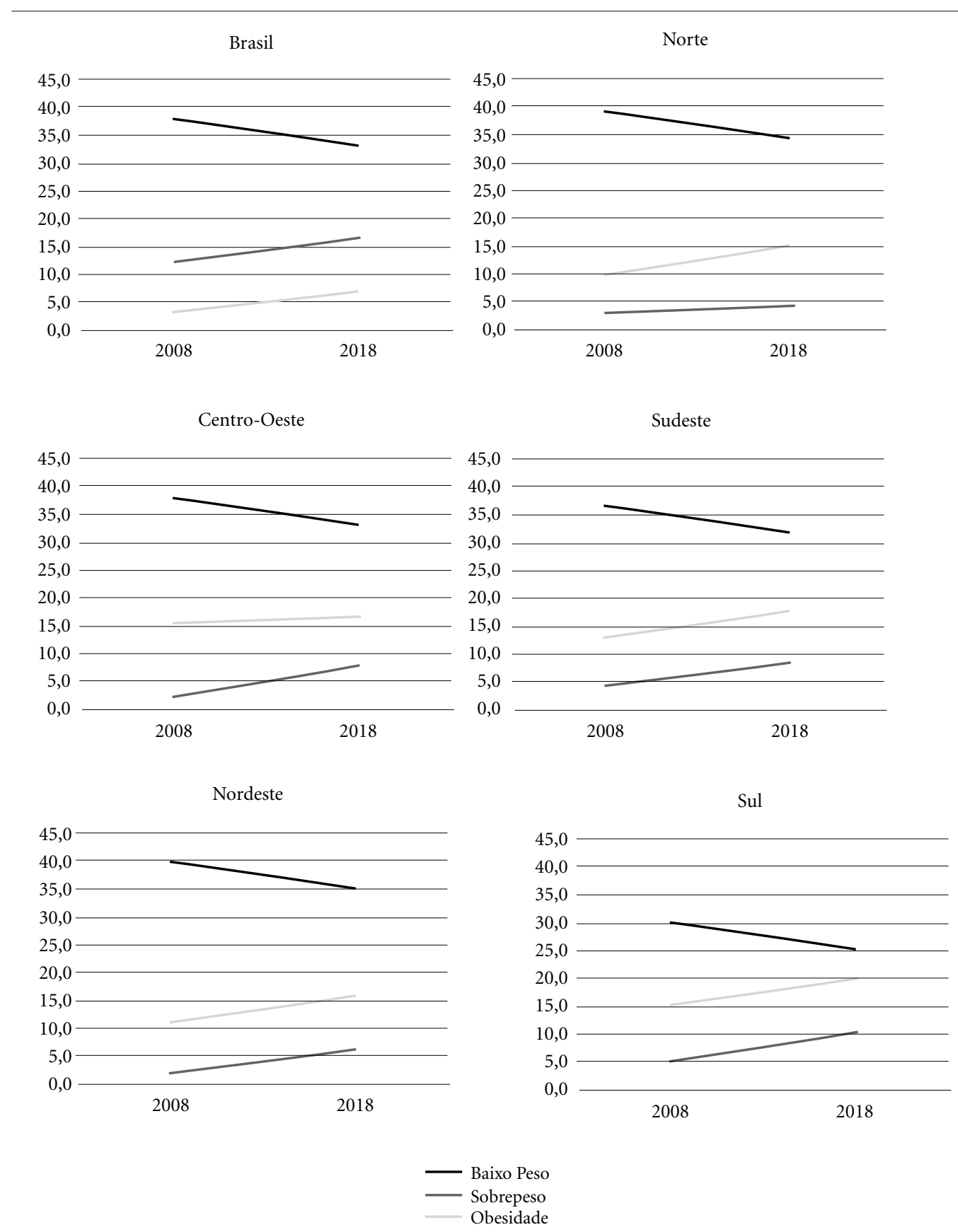

Figura 1. Prevalências brutas de baixo peso, sobrepeso e obesidade em gestantes adolescentes beneficiárias do Programa Bolsa Família nos anos 2008 e 2018, segundo macrorregiões do Brasil.

Fonte: Sistema de Vigilância Alimentar e Nutricional Web.

os desvios nutricionais nesta fase $\mathrm{e}^{29,30}$. Um estudo realizado com as gestantes adolescentes e adultas que estavam em acompanhamento pré-natal em uma Unidade Básica de Saúde no Rio Grande do Sul buscou investigar o impacto de um programa de orientação dietética no controle de ganho de peso gestacional, e observou que este foi eficaz em diminuir o ganho de peso e em reduzir intercorrências clínicas como diabetes gestacional, pré-eclâmpsia, baixo peso e prematuridade ${ }^{31}$. 
Tabela 2. Número de gestantes localizadas por equipes de saúde e percentual de gestantes com dados nutricionais incluídos nos sistemas de informação em saúde por macrorregiões do Brasil, em 2008 e 2018.

\begin{tabular}{lcrrrrr}
\hline \multirow{2}{*}{ Local } & \multicolumn{3}{c}{$\mathbf{2 0 0 8}$} & \multicolumn{2}{c}{$\mathbf{2 0 1 8}$} \\
\cline { 2 - 7 } & $\begin{array}{c}\text { Gestantes } \\
\text { localizadas }\end{array}$ & $\begin{array}{c}\text { Gestantes com dados } \\
\text { nutricionais }\end{array}$ & $\begin{array}{c}\text { Gestantes } \\
\text { localizadas }\end{array}$ & $\begin{array}{c}\text { Gestantes com dados } \\
\text { nutricionais }\end{array}$ \\
\cline { 2 - 7 } & $\mathbf{n}$ & $\mathbf{n}$ & $\mathbf{9}$ & $\mathbf{n}$ & $\mathbf{n}$ & \% \\
\hline Norte & 9.946 & 6.648 & 66,8 & 66.916 & 60.057 & 89,8 \\
Nordeste & 42.006 & 24.488 & 58,3 & 225.289 & 194.129 & 86,2 \\
Centro-Oeste & 4.455 & 2.396 & 53,8 & 24.424 & 20.457 & 83,8 \\
Sudeste & 25.945 & 12.164 & 46,9 & 130.758 & 116.678 & 89,2 \\
Sul & 10.572 & 5.584 & 52,8 & 36.795 & 31.246 & 84,9 \\
Brasil & 92.924 & 51.280 & 55,2 & 484.182 & 422.567 & 87,3 \\
\hline
\end{tabular}

Fonte: Sistema Bolsa Família na Saúde e Sistema Bolsa Família.

Gestantes adolescentes por si só compõem um grupo vulnerável para desvios nutricionais e isso se dá por diversos motivos como a demanda aumentada por nutrientes para subsidiar o crescimento e desenvolvimento materno e da prole no ambiente intrauterino, e hábitos alimentares inadequados. Nesta perspectiva, não é incomum a presença de desvios nutricionais nesta população. Semelhante aos nossos achados, na Colômbia, um estudo realizado com 294 gestantes adolescentes, onde $80 \%$ destas estavam nos mais baixos estratos socioeconômicos, encontrou $33,7 \%$ de gestantes com baixo peso e $14,3 \%$ com excesso de peso $^{32}$. Diferentemente, dados de uma pesquisa realizada no Rio de Janeiro, evidenciou que cerca de $13 \%$ das puérperas adolescentes atendidas na maternidade de um hospital público iniciaram a gestação com algum desvio nutricional, sendo $1,0 \%$ baixo peso e $12,0 \%$ excesso de peso $^{33}$.

A gestação na adolescência parece trazer desfechos desfavoráveis, onde o nascimento prétermo é um dos principais, sendo este mais frequente nas adolescentes menores de 15 anos, segundo Karatasli et al. ${ }^{34}$, que assim como em outro estudo realizado na Turquia encontrou uma alta taxa de internação na unidade de terapia intensiva neonatal de proles destas adolescentes ${ }^{35}$. Além disso, a gestação na adolescência parece trazer repercussões negativas para o crescimento da adolescente, como mostrado por Rah et al. ${ }^{7}$, onde as gestantes adolescentes tiveram uma diferença de altura de $0,35 \pm 0,85 \mathrm{~cm} /$ ano em relação às adolescentes que nunca engravidaram. Este déficit estatural associado a ossos pélvicos subdesenvolvidos e, portanto, uma pélvis contraída, pode explicar o aumento do risco de gestantes adolescentes ne- cessitarem realizar o parto cesáreo ${ }^{34,36}$.

Métodos para avaliação antropométrica de gestantes são frágeis, principalmente ao se levar em consideração que os instrumentos desenvolvidos pelo Institute of Medicine $e^{37,38}$ e o nomograma de Atalah et al. ${ }^{20}$ foram escassamente validados nos países da América Latina, o que nos sugere cautela no processo de comparabilidade a contextos epidemiológicos com fenótipos e condições socioambientais distintos ${ }^{20,37-41}$. O nomograma de Atalah et al. ${ }^{20}$ pode levar a interpretações de alta prevalência para o diagnóstico de déficit nutricional/baixo peso e uma menor sensibilidade para identificar casos de sobrepeso/ obesidade devido à sua faixa de IMC adequada na $10^{\text {a }}$ semana gestacional $\left(20,3\right.$ a $\left.25,2 \mathrm{~kg} / \mathrm{m}^{2}\right)$, quando comparado com os demais métodos de avaliação antropométrica de gestantes ${ }^{42}$.

A falta de um padrão ouro existente para definir o excesso de peso e baixo peso na gestação, dificulta a realização de estudos de acurácia diagnóstica dos instrumentos baseados na relação peso-estatura, principalmente pela ausência de curvas específicas para a avaliação de gestantes adolescentes. Desta forma, a preocupação na avaliação de gestantes adolescentes se agrava ainda mais, uma vez que se refere à convergência de dois momentos nutricionais e metabolicamente críticos, a gestação e a fase da adolescência ${ }^{43,44}$.

Este estudo possui algumas limitações que devem ser levadas em consideração na apreciação dos resultados apresentados. Primeiramente, os dados de cobertura das condicionalidades de saúde e, consequentemente, o percentual de gestantes que possuíam dados nutricionais incluídos nos sistemas de informações são dados para gestantes em qualquer faixa etária, e não es- 
pecíficos para as gestantes adolescentes. Este fato pode subestimar ou superestimar o percentual de gestantes adolescentes que tiveram os seus dados incluídos no sistema. Outra limitação que deve ser levada em consideração é que as prevalências foram geradas a partir das observações registradas no Sistema de Gestão do PBF na Saúde do Ministério da Saúde, sendo uma amostra do universo de gestantes adolescentes beneficiárias do PBF, não permitindo ser extrapolado para gestantes adolescentes não beneficiárias do programa. Além disto, algumas limitações apresentadas pelo SISVAN devem ser consideradas. O sistema gera relatórios de dados e informações referentes ao estado nutricional a partir do ano 2008, o que impede a avaliação de forma integral das tendências do estado nutricional desde o início do PBF na população beneficiária. Finalmente, a ferramenta utilizada para a classificação do estado nutricional pelo PBF não foi desenvolvida para a população de gestantes adolescentes, como discutido anteriormente, porém é a ferramenta indicada pelo Ministério da Saúde para acompanhamento das gestantes nesta faixa etária.

Como pontos fortes, destaca-se que os dados representam o panorama nacional do estado nutricional das gestantes adolescentes beneficiárias do PBF, ou seja, todos os estados e macrorregiões foram representados neste estudo. Além disso, desconhecemos estudos que tiveram o objetivo de determinar a tendência da prevalência de desvios nutricionais nesta população numa avaliação a nível nacional, o que torna este o primeiro estudo a traçar o cenário nacional destas condições em gestantes adolescentes beneficiárias do PBF.

\section{Conclusão}

Conclui-se que a prevalência de baixo peso entre as gestantes adolescentes beneficiárias do PBF apresentou uma tendência decrescente em todas as regiões do Brasil, ao passo que as prevalências de sobrepeso e obesidade apresentam uma tendência crescente em todo o país no período. Por fim, destaca-se a importância de estudos com métodos capazes de identificar os determinantes do fenômeno da gestação na adolescência e da mudança no perfil nutricional nesta população para subsidiar a formulação de políticas públicas direcionadas para esta condição, tendo em vista as repercussões dos desvios nutricionais nesta fase do ciclo da vida e os desfechos desfavoráveis da gestação. 


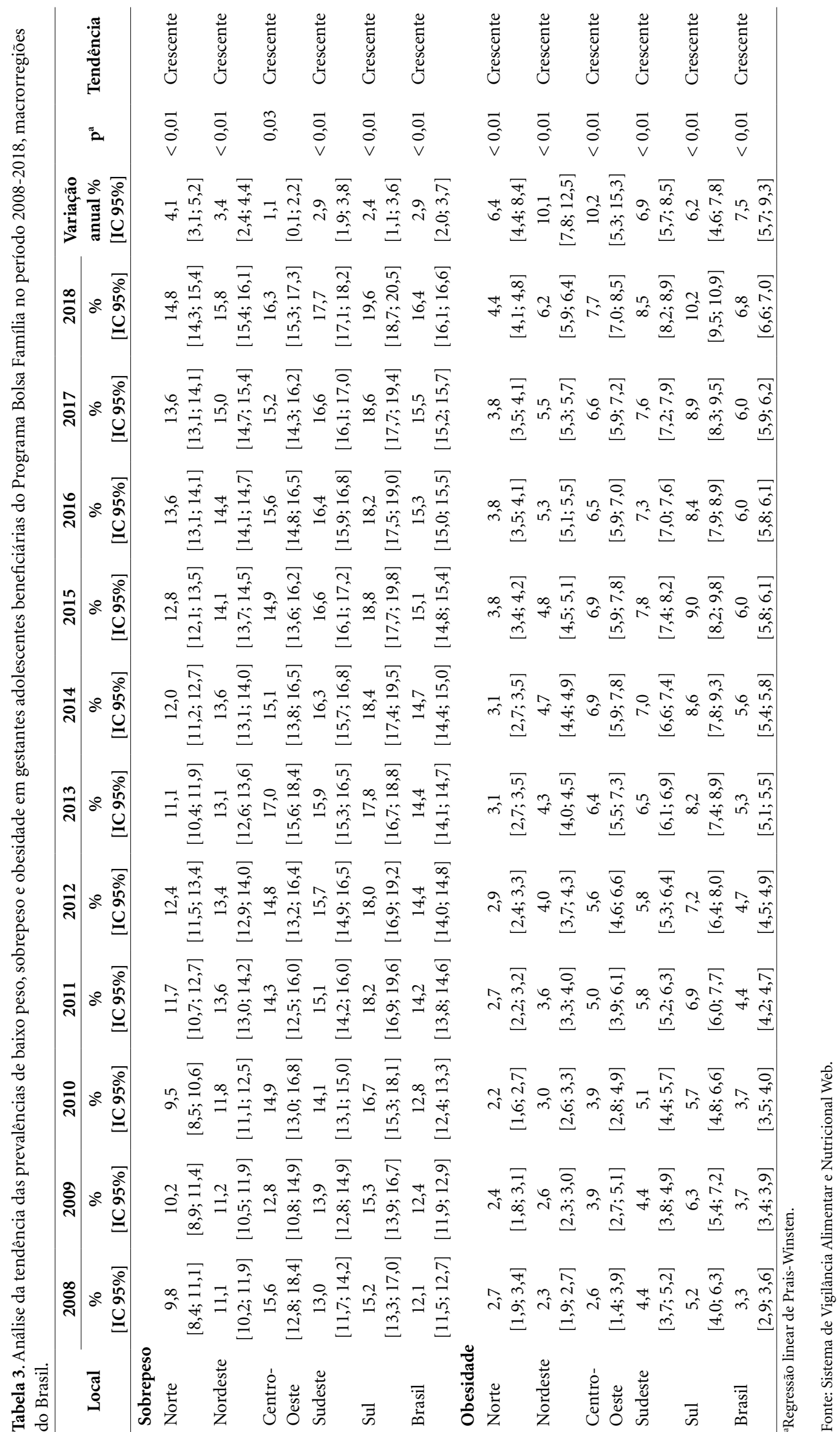




\section{Colaboradores}

AE Silva Júnior participou da concepção do estudo, construção do banco de dados, análise e interpretação dos dados, redação do artigo e revisão crítica do conteúdo. ML Macena participou da construção do banco de dados, análise e interpretação dos dados, redação do artigo e revisão crítica do conteúdo. LGL Vasconcelos, NB Almeida, DRS Praxedes, IROM Pureza, NB Bueno e APG Clemente participaram da redação do artigo e revisão crítica do conteúdo. Todos os autores leram e aprovaram a versão final do conteúdo.

\section{Referências}

1. United Nations Population Fund (UNFPA). Motherhood in childhood: facing the challenge of adolescent pregnancy. New York: UNFPA; 2013.

2. World Health Organization (WHO). Young people's health: a challenge for society. Geneva: WHO; 1986.

3. World Health Organization (WHO). WHO Guidelines on Preventing Early Pregnancy and Poor Reproductive Outcome Among Adolescents in Developing Countries. Geneva: WHO; 2011.

4. Rodríguez Vignoli J. La reproducción en la adolescencia y sus desigualdades en América Latina: introducción al análisis demográfico, con énfasis en el uso de microdatos censales de la ronda de 2010. Santiago: Comisión Económica para América Latina y el Caribe; 2014.

5. Pan American Health Organization (PAHO). United Nations Population Fund and United Nations Children's Fund (UNICEF). Accelerating progress toward the reduction of adolescent pregnancy in Latin America and the Caribbean. Report of a technical consultation. Washington D.C.: PAHO, UNICEF; 2016.

6. Gigante DP, Rasmussen KM, Victora CG. Pregnancy increases BMI in adolescents of a population based birth cohort. J Nutr 2005; 135(1):74-80.

7. Rah JH, Christian P, Shamim AA, Arju UT, Labrique AB, Rashid M. Pregnancy and Lactation Hinder Growth and Nutritional Status of Adolescent Girls in Rural Bangladesh. J Nutr 2008; 138(8):1505-1511.

8. Azevedo WF, Diniz MB, Fonseca ESV, Azevedo LMR, Evangelista CB. Complicações da gravidez na adolescência: revisão sistemática da literatura. Einstein 2015; 13(4):618-626.

9. Ganchimeg T, Ota E, Morisaki N, Laopaiboon M, Lumbiganon P, Zhang J, Yamdamsuren B, Temmerman M, Say L, Tunçalp O, Vogel JP, Souza JP, Mori R, WHO Multicountry Survey on Maternal Newborn Health Research Network. Pregnancy and childbirth outcomes among adolescent mothers: a world health organization multi country study. BJOG 2014; 121(Supl. 1):40-48.

10. Nguyen PH, Sanghvi T, Tran LM, Afsana K, Mahmud Z, Aktar B, Haque R, Menon P. The nutrition and health risks faced by pregnant adolescents: insights from a cross -sectional study in Bangladesh. PLoS One 2017, 12(6):e0178878.

11. Santelli JS, Song X, Garbers S, Sharma V, Viner RM. Global trends in adolescent fertility, 1990-2012, in relation to national wealth, income inequalities, and educational expenditures. I Adolesc Health 2017; 60(2):161-168

12. Gertler P. Final report: the impact of PROGRESA on health. Washington, DC: Food Policy Research Institute; 2000.

13. Soares FV. Brazil's Bolsa Família: a review. Econ Polit Weekly 2011; 46:55-60.

14. Shei A, Costa F, Reis MG, Ko AI. The impact of Brazil's Bolsa Família conditional cash transfer program on children's health care utilization and health outcomes. BMC Int Health Hum Rights 2014; 14:1-10. 
15. Controladoria Geral da União. Portal da Transparência [Internet]. [acessado 2020 fev 10]. Disponível em: http://www.portaltransparencia.gov.br.

16. Brasil. Ministério da Saúde (MS). Secretaria de Atenção à Saúde. Departamento de Atenção Básica. Marco de referência da vigilância alimentar e nutricional na atenção básica. Brasília: MS; 2015.

17. Brasil. Ministério do Desenvolvimento Social. Secretaria de Avaliação e Gestão da informação. Manual do pesquisador. Brasília: Ministério do Desenvolvimento Social, Programa Bolsa Família; 2018.

18. Cotta RMM, Machado JC. Programa Bolsa Família e segurança alimentar e nutricional no Brasil: revisão crítica da literature. Rev Panam Salud Publica 2013; 33(1):54-60.

19. Lignani JB, Sichieri R, Burlandy L, Salles-Costa R. Changes in food consumption among the Programa Bolsa Família participant families in Brazil. Public Health Nutr 2010; 14:785-792.

20. Atalah SE, Castillo LC, Castro SR, Aldea A. Propuesta de un nuevo estándar de evaluación nutricional en embarazadas. Rev Med Chil 1997; 125(12):1429-1436.

21. Brasil. Ministério da Saúde (MS). Secretaria de Atenção à Saúde. Departamento de Atenção Básica. Orientações para coleta e análise de dados antropométricos em serviços de saúde: norma técnica do Sistema de Vigilância Alimentar e Nutricional - SISVAN. Brasília: MS; 2011.

22. Antunes JLF, Cardoso MRA. Uso da análise de séries temporais em estudos epidemiológicos. Epidemiol Serv Saude 2015; 24(3):565-576.

23. Pinto LF, Giovanella L. Do Programa à Estratégia da Saúde da Família; expansão do acesso e redução das internações por condições sensíveis à atenção básica (ICSAB). Cien Saude Colet 2018; 23(6):1903-1914.

24. Seidell JC, Halberstadt J. The Global Burden of Obesity and the Challenges of Prevention. Ann Nutr Metab 2015; 66(Supl. 2):7-12.

25. Popkin BM, Corvalan C, Grummer-Strawn LM. Dynamics of the double burden of malnutrition and the changing nutrition reality. Lancet 2020; 395:65-74.

26. Gray CL, Messer LC, Rappazo KM, Jyotsna SJ, Grabich SC, Lobdell DT. The association between physical inactivity and obesity is modified by five domains of environmental quality in U.S. adults: A cross-sectional study. PLoS One 2018; 13(8):e0203301.

27. Wells JC, Sawaya AL, Wibaek R, Mwangome M, Poullas MS, Yajnik CS, Demaio. The Double Burden of Malnutrition: Aetiological Pathways and Consequences for Health. Lancet 2019; 395(10217):75-88.

28. Catalano PM, Shankar K. Obesity and pregnancy: mechanisms of short term and long term adverse consequences for mother and child. BMJ 2017; 356:j1.

29. Brasil. Ministério da Saúde (MS). Secretaria de Atenção à Saúde. Departamento de Atenção Básica. Manual Instrutivo das Ações de Alimentação e Nutrição na Rede Cegonha. Brasília: MS; 2013.
30. Niquini RP, Bittencourt SA, Lacerda, EMA, Saunders C, Leal, MC. Evaluation of the prenatal nutritional care process in seven family health unit in the city of Rio de Janeiro. Cien Saude Colet 2012; 17(10):28052816.

31. Vitolo MR, Bueno MSF, Gama CM. Impacto de um programa de orientação dietética sobre a velocidade de ganho de peso de gestantes atendidas em unidades de saúde. Rev Bras Ginecol Obstet 2011; 33(1):13-19.

32. Restrepo-Mesa SL, Zapata NL, Parra BES, Escudero LEV, Betancur LA. Estado nutricional materno y neonatal en un grupo de adolescentes de la ciudad de Medellín. Nutr Hosp 2015; 32(3):1300-1307.

33. Santos MMADS, Baião MR, Barros DCD, Pinto ADA Pedrosa PLM, Saunders C. Estado nutricional prégestacional, ganho de peso materno, condições da assistência pré-natal e desfechos perinatais adversos entre puérperas adolescentes. Rev Bras Epidemiol 2012; 15(1):143-154.

34. Karatasli V, Kanmaz AG, Inan AH, Budak A, Beyan E. Maternal and neonatal outcomes of adolescent pregnancy. J Gynecol Obstet Hum Reprod 2019; 48(5):347350.

35. Kirbas A, Gulerman HC, Daglar K. Pregnancy in Adolescence: Is it an obstetrical risk? J Pediatr Adolesc Gynecol 2016; 29(4):367-371.

36. Shirima CP, Kinabo JL. Nutritional status and birth outcomes of adolescent pregnant girls in Morogoro, Coast, and Dar es Salaam regions, Tanzania. Nutrition 2005; 21(1):32-38.

37. Institute of Medicine. Full-sized BMI and weight gain charts. Supplementary materials for nutrition during pregnancy and lactation: an implementation guide. Washington, DC: National Academy Press; 1992.

38. Institute of Medicine, National Research Council. Committee to Reexamine IOM Pregnancy Weight Guidelines; Rasmussen KM, Yaktine AL, editores. Weight gain during pregnancy: reexamining the guidelines. Washington, DC: National Academies Press; 2009.

39. Coelho KS, Souza AI, Batista Filho M. Avaliação antropométrica do estado nutricional da gestante: visão retrospectiva e prospectiva. Rev Bras Saude Mater Infant 2002; 2(1):57-61.

40. Barros DC, Saunders C, Leal C. Avaliação nutricional antropométrica de gestantes brasileiras: uma revisão sistemática. Rev Bras Saude Mater Infant 2008; 8(4):363-376.

41. Padilha PDC, Accioly E, Veiga GV, Bessa TC, Libera BD. Nogueira JL, Alves PD, Souza Junior PR, Saunders C. The performance of various anthropometric assessment methods for predicting low birth weight in pregnant women. Rev Brasil Saude Mater Infant 2009; 9(2):197-206.

42. Silva SL, Bresani-Salvi C, Caminha MFC, Figueroa JN, Batista Filho M. Classificação antropométrica de gestantes: comparação entre cinco métodos diagnósticos utilizados na América Latina. Rev Panam Salud Publica 2017; 41:e85. 
43. Canavan CR. Fawzi WW. Addressing Knowledge Gaps in Adolescent Nutrition: Toward Advancing Public Health and Sustainable Development. Curr Dev Nutr 2019; 3:7.

44. Das JK, Salam RA, Thornburg KL, Prentice AM, Campisi S, Lassi ZS, Koletzko B, Bhutta ZA. Nutrition in adolescents: physiology, metabolism, and nutritional needs. Ann NY Acad Sci 2017; 1393(1):21-33.

Artigo apresentado em 13/03/2020

Aprovado em 08/04/2021

Versão final apresentada em 10/04/2021

Editores-chefes: Romeu Gomes, Antônio Augusto Moura da Silva 